\title{
OPERATING MODES FOR COMPULSATOR BASED ELECTROMAGNETIC LAUNCHER SYSTEMS
}

By:

S.B. Pratap

J.P. Kajs

W.A. Walls

W.F. Weldon

J.R. Kitzmiller

S.K. Murthy

Digest of Technical Papers of the 10th IEEE International Pulsed Power Conference, July 3-6, 1995, vol. 1, pp. 180-185

PN - 226

Center for Electromechanics

The University of Texas at Austin

PRC, Mail Code R7000

Austin, TX 78712

(512) 471-4496 


\title{
OPERATING MODES FOR COMPULSATOR BASED ELECTROMAGNETIC LAUNCHER SYSTEMS
}

\author{
S. B. Pratap, J.P. Kajs, W.A. Walls, W.F. Weldon, J.R. Kitzmiller \\ Center for Electromechanics \\ The University of Texas at Austin \\ PRC, MC R7000 \\ Austin, TX 78712
}

(512) $471-4496$

\author{
S. K. Murthy \\ Delphi Saginaw Steering Systems \\ 3900 Holland Road \\ Saginaw, Michigan 48601
}

\begin{abstract}
The compensated pulsed alternator (compulsator) is a versatile power supply capable of interfacing with the electromagnetic launcher in various ways. The method that has been explored at length with several systems is the single phase option. Several variants of this option, some using advanced pulse shaping techniques, have been discussed in prior publications $[1-3]$.

Besides this basic single pulse method of operating there are several other methods each with its pros and cons. The multi-phase option is discussed in this paper. Within the broad class of multi-phase systems there are further sub-classes, namely alternating current drive and unidirectional current drives. Thus the branching of these operating modes gives rise to a variety of operating modes. Each one of these operating modes is described and simulation results are presented.
\end{abstract}

\section{Introduction}

Compulsator driven electromagnetic (EM) gun systems built and tested to date have been of the single phase variety. The single phase system has performed excellently when driving EM guns and with the help of appropriately oriented compensating windings are capable delivering an optimally shaped current pulse to the gun. The drawback of the single phase system especially for large (multi-megajoule systems) is that the electrical period of the machine needs to be approximately equal to the pulse width of the load pulse. This requirement results in machines with a minimum number of poles - preferably 2 poles. The lower the number of poles for a given pulse width, the higher the rotational speed, and therefore lower the moment inertia for a given energy storage. Thus for single phase machines lower poles imply higher energy density. There are several reasons why a 2 pole machine is not ideal for aspects other than energy storage. The external magnetic fields are higher and decay slower with distance. The end turns of the windings are usually bulkier and the armature winding is difficult to fabricate because it is radially thin and azimuthally wide. Transposition of the conductors becomes difficult. These and several other difficulties of two pole machines must be overcome and even then, there is an upper limit to the rotational speed. Due to these drawbacks alternate schemes have been investigated and acceptable solutions have been found for high energy systems. In these newer systems the pulse width and machine rotational speeds have been decoupled thus allowing independent selection of the number of poles and rpm.

\section{Single Phase System}

The single phase mode (fig. 1) consists of a single phase compulsator driving a two rail railgun. The pulse shape is tailored by using the selectively passive compensation technique. The pulse width determines the operating frequency of the machine. It is possible to achieve a good acceleration ratio (average to peak value of 0.6 or better) with the selective passive machine. Figure 2 illustrates a pulse shape for a typical mission using a selective passive compulsator. The operating voltage is around $6 \mathrm{kV}$. With the single phase mode, the jerk on the projectile is lower than that in the other operating modes. The gun
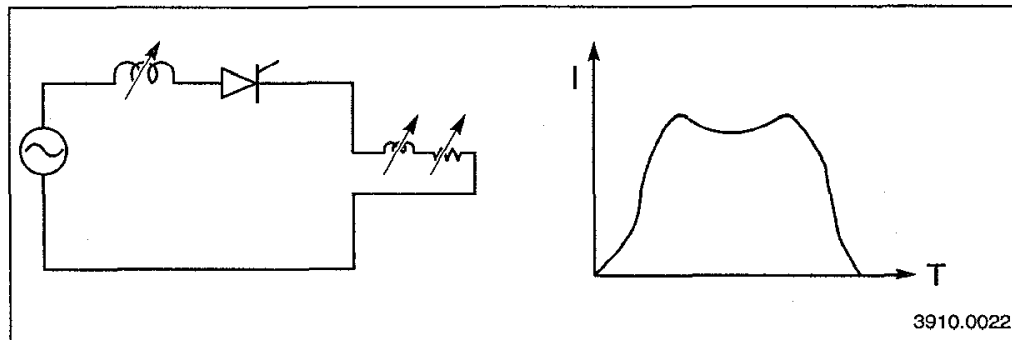

Figure 1. Single phase mode of operation muzzle current, however, is high if acceleration ratios $>0.6$ are needed. Any attempt to lower the exit current affects the acceleration ratio in an adverse manner. A muzzle switch and resistor would help to overcome this drawback.

There are in essence only two control parameters for the single phase mode, i.e. the firing angle on the machine open circuit voltage cycle and the field current of 


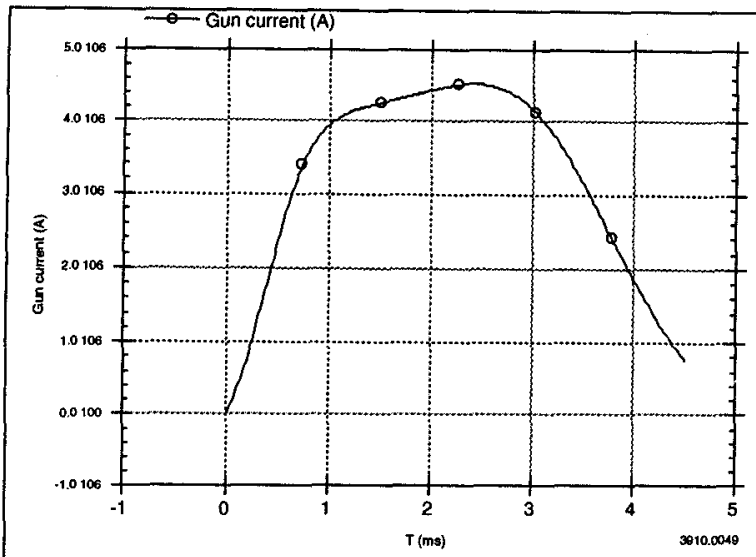

Figure 2. Current pulse from a selective passive machine

the gun switch, which can be varied from shot to shot to affect the performance of the single phase selective passive machine. The orientation of the compensating winding which plays a major role in determining the pulse shape is fixed in the present instance. Hence the single phase mode has limited flexibility.

The duty on the closing switch is mild compared to the staged discharge mode and the switch does not have to commutate current. The operating voltage of the machine is lower than the other modes of operation (due to the low frequency and the dc pulse mode of operation).

\section{Multiphase Staged Discharge Mode (or Half Wave Rectified)}

The staged discharge mode uses a multiphase passive compulsator and operates at a higher frequency than the single phase selective passive machine and is hence potentially lower in weight. The pulse shaping is achieved by switching in phases sequentially so that a dc pulse is obtained in the EM gun. Each phase has a switch in series with it and hence conducts only in one direction. Figure 3 is a schematic representation of the staged discharge mode of operation. The staged discharge scheme feeds a two rail railgun with a return path through the neutral.

Another variation of the staged discharge mode which uses fewer switches is shown by figure 4 . This scheme uses four rails (for a two phase system) and one phase is connected across each rail pair. The scheme is described below in greater detail. In general the jerk on the projectile is higher than the single phase mode. The projectile also suffers a higher jerk during current commutation. The switches have to be designed for the commutation duty.

There are several advantages of this operating mode. Since each phase is endowed with a switch, there is considerable flexibility in controlling the shape of the pulse. The gun muzzle current can be minimized without significantly affecting the acceleration ratio. Staged discharge mode of operation can be achieved with two or more phases. The control and flexibility improve with the number of phases.

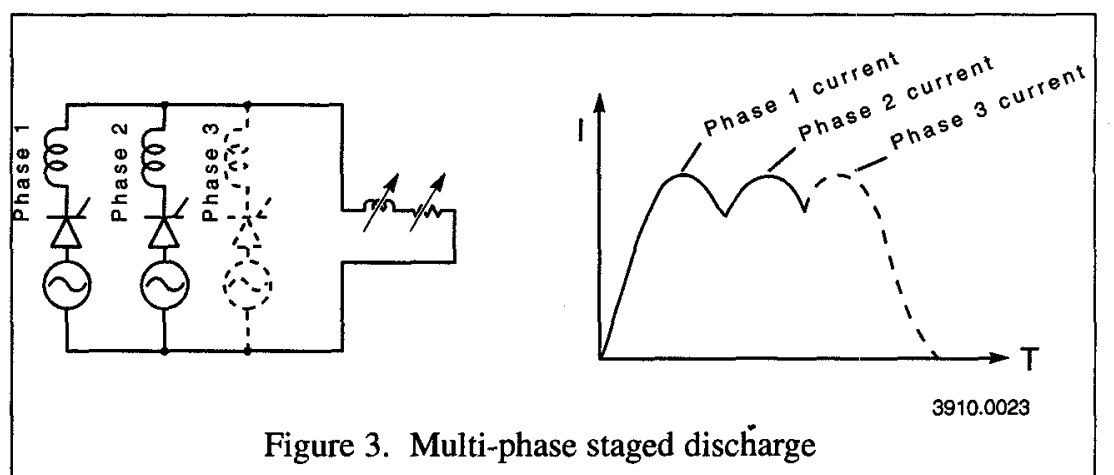

In the case of the two phase machine, the phase angle between the phases is not conducive for switching in the first phase again after the second phase. Thus for two phase systems there is a link between the pulse width and machine rotational speed. For the three phase machine the first phase can to be designed for the voltage required to supply the gun current towards the end of the launch. In such a situation, it is beneficial to design all of the three phases for the same voltage,

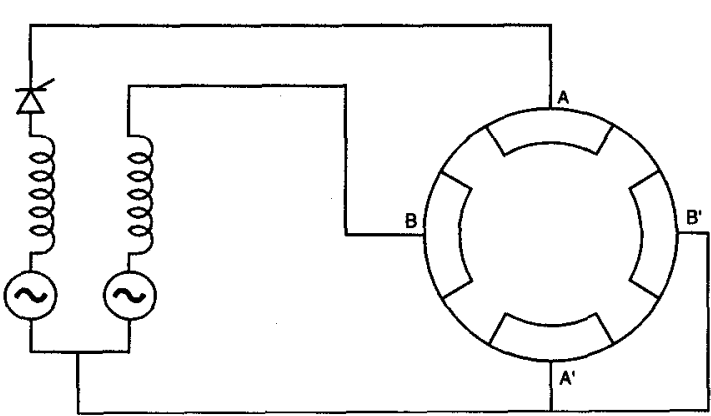

3910.0030

Figure 4. Two phase, four rail staged discharge scheme implying a balanced three phase system. The ability to repeatedly switch the phases, allows the multiphase system the potential to be used for a variety of missions (varying gun lengths, projectile kinetic energy etc.), thus providing significant flexibility.

A potential problem with operating at high frequencies in the staged discharge mode is the inability to extract the magnetic energy out of the gun at exit. The phase voltage and frequency (i.e. the flux or the volt-seconds) should be high enough to absorb the magnetic flux in the rails at the end of the launch. If the higher voltage is undesirable a muzzle resistor can be included so that gun 


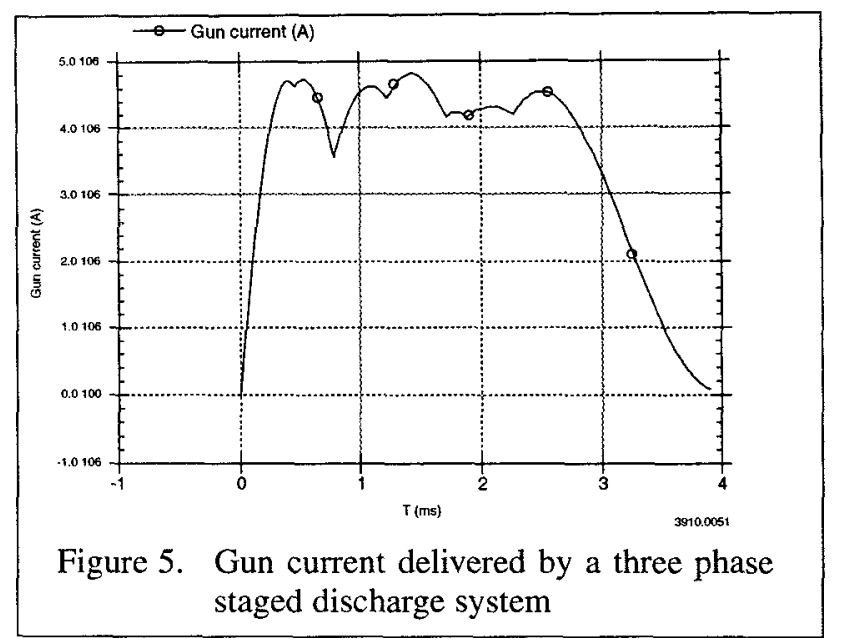

The phase with the lower voltage is switched on and it drives the projectile down the rails. The second phase is directly connected to the staggered rails. So when the projectile arrives at the location of the second pair of rails, the second phase starts conducting and drives the projectile till exit. The first pair of rails can be made long enough to ensure zero current in phase 1. Unlike the two phase staged discharge two rail railgun system, the second set of rails in the four rail system do not carry any current till the injection of the projectile, hence the second phase has to drive the current up from zero to peak resulting in a higher voltage (by $20 \%$ compared to the two rail railgun) requirement. The system has the advantages of fewer switches and the switches do not need to commutate the current as in the two phase staged discharge system. However, the system has poor control and flexibility resulting in poor acceleration ratios.

\section{Multiphase Full Wave Rectified Mode}

The staged discharge mode is similar to half wave rectification. If the output of the compulsator is fully rectified, then a dc pulse can be obtained to drive the railgun. The full wave rectifier however needs a greater number of switches compared to the staged discharge mode. At any given instant during a discharge all the machine phases are conducting. Hence the phases and the switches need to be designed for the additional action. Figure 6 is a circuit representation of the full wave rectified mode.

The full wave rectified mode has the potential advantage of more control and flexibility than the staged discharge mode. Better control over the pulse shape and the acceleration ratio is attained. All three phases of the machine are repeatedly switched in and out throughout the launch. The number of switching operations and therefore commutations is also higher when using full wave rectification. The result is that the machine spends a good portion of the launch time commutating rather than driving the load. The machine correspondingly needs a higher voltage since the volt-seconds must be delivered in a shorter time.

\section{Multiphase ac Mode}

The ac railgun systems which have been examined all consist of multi-phase alternators and multi-rail railguns. The distinguishing characteristic of an ac railgun is that it uses more than one pulse from the power supply and drives the current through zero and negative during the launch for each of the pulses. The multiple rails and phases are

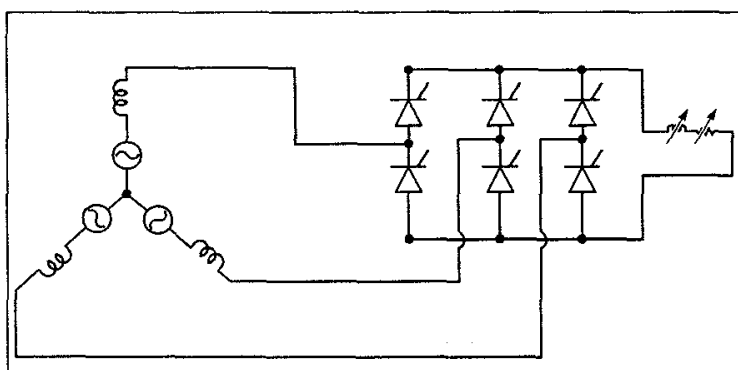

Figure 6. Three phase, full wave rectified scheme

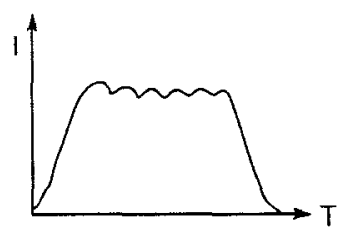

3910.0024 required to maintain a fairly constant acceleration. The main difficulty with an alternating current railgun is that as the package accelerates the impedance of the launcher increases. The increasing impedance of the launcher decreases the launcher current and acceleration produced by the later pulses. The acceleration tends to have a peak at the first pulse and decrease for each succeeding pulse. 

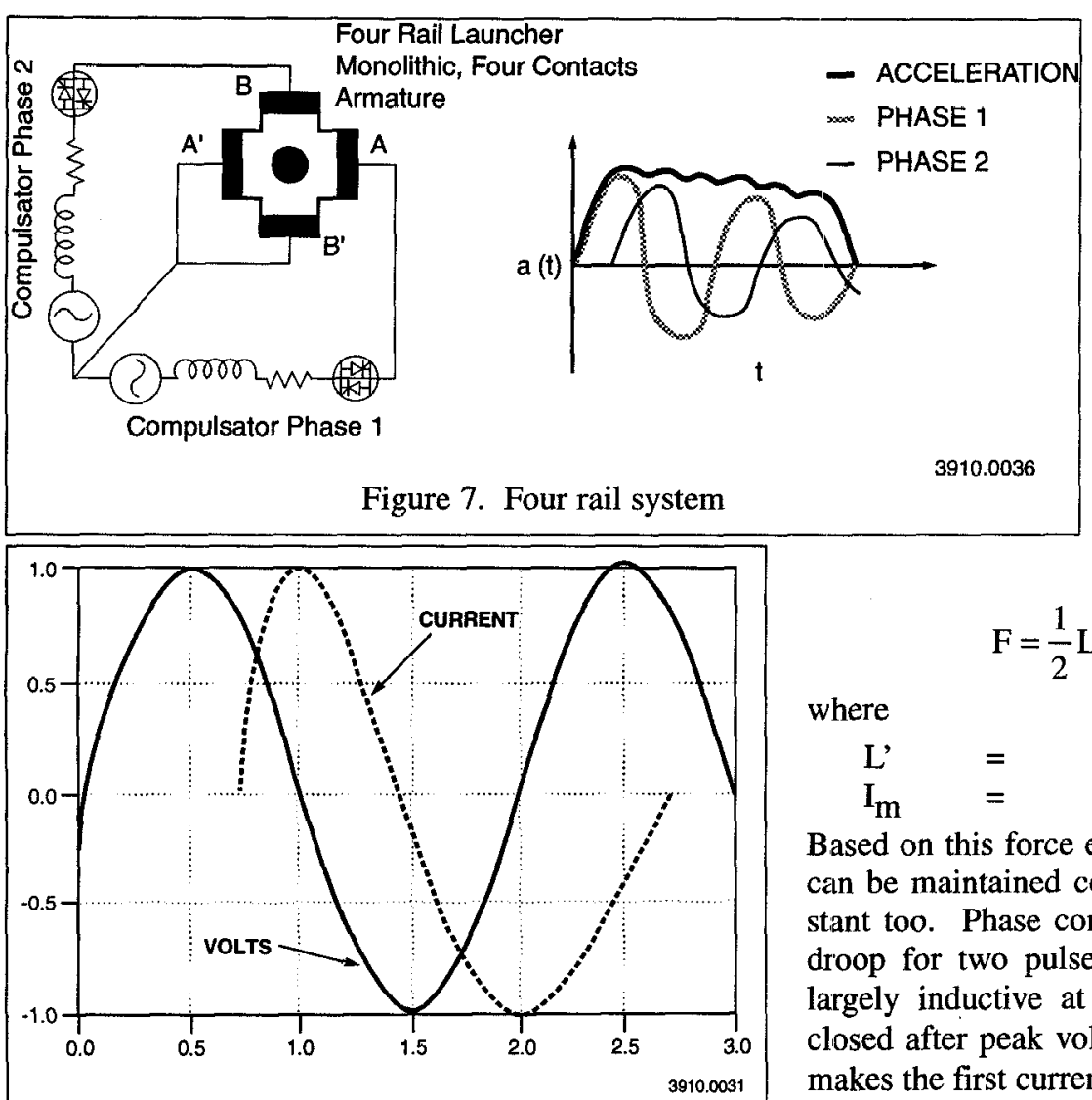

Figure 8. Example of effect of phase control for ac railgun

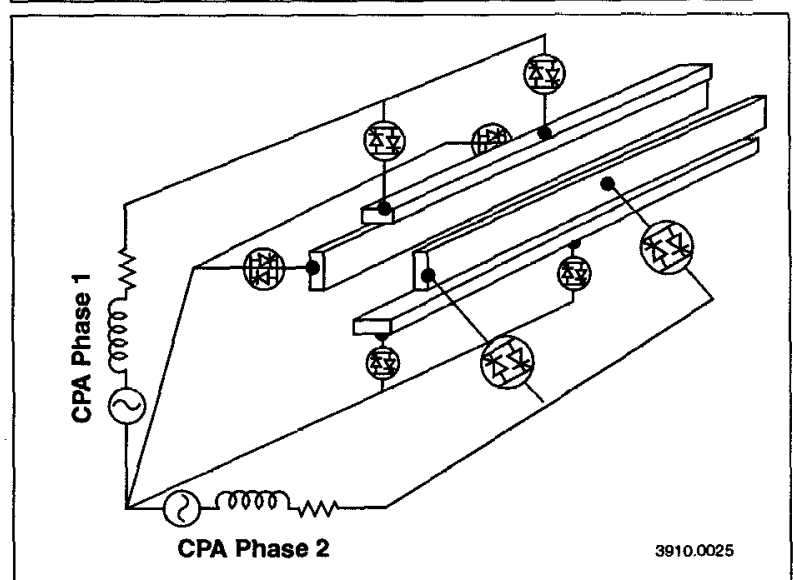

Figure 9. AC drive system multiple feed railgun

where ilar to the first pulse.

\section{Two Phase ac Railgun}

The two phase system uses four rails. The four rail system is shown in figure 7. One phase is connected across the two rails A-A' and the second phase is connected across rails B-B'. The two phase currents can be described as,

$$
\begin{aligned}
& \mathrm{I}_{\mathrm{a}}=\mathrm{I}_{\mathrm{m}} \sin (\omega \mathrm{t}) \\
& \mathrm{I}_{\mathrm{b}}=\mathrm{I}_{\mathrm{m}} \cos (\omega \mathrm{t})
\end{aligned}
$$

The force on the projectile is given by

$$
F=\frac{1}{2} L^{\prime} I_{m}^{2}=\frac{1}{2} L^{\prime \prime}\left(I_{a}^{2}+I_{b}^{2}\right)
$$

$\mathrm{L}^{\prime} \quad=\quad$ inductance gradient of the gun $(\mathrm{H} / \mathrm{m})$

$\mathrm{I}_{\mathrm{m}} \quad=\quad$ peak current per phase (A)

Based on this force equation it is clear if the peak current can be maintained constant then the force would be constant too. Phase control can be used to help the current droop for two pulses in succession. The impedance is largely inductive at higher frequencies. The switch is closed after peak voltage for the first current pulse. This makes the first current pulse shorter and smaller than if the switch were closed at zero voltage. The switch remains closed or is closed again as the current crosses zero. The second pulse is longer than the first pulse. Because of the increased gun impedance this second pulse has the same peak current as the first pulse though of a different sign. An illustration of the open circuit voltage and current shapes for a two pulse burst is shown in figure 8. The third pulse would have a significantly lower peak current since it would have a still higher impedance and a short pulse sim-

Active impedance control can be used to slow the increase in gun impedance. The most direct method is to use a distributed current feed railgun. A distributed current feed railgun is identical to a distributed energy store (DES) railgun except that multiple power supplies are not required. The current feeds into the railgun are spaced such that the current crosses through zero just before the armature passes a current feed. A low impedance buswork with a switch connects the multiple feeds to the breech of the gun. The switches are closed for each of the current feeds as current crosses zero after the armature passes them. Figure 9 illustrates this concept. In this way the impedance of the railgun is maintained approximately a constant. The main difficulty with this scheme is that the buswork connections to the rails are non-trivial and are detrimental to the structure around the rails. The second problem associated with this scheme is that the low impedance buswork must be moved with the gun and will tend to be heavy.

One means around most of the problems associated with the distributed current feed launcher is to add a set of switched anti-augmenting rails. These anti-augmenting rails only run part of the length of the gun and are switched into the circuit after the armature passes just like the distributed current feed launcher. Figure 10 illustrates this concept. There are several variations in implementing this concept. It is possible to use switched shorted turns rather than series con- 
nected anti-augmenting rails. Electrically the anti-augmented launcher is very similar to the distributed current feed launcher. The main difference is that the resistance for the anti-augmented launcher will probably be higher than the distributed current feed launcher. However the anti-augmenting launcher should be lighter and have a better structure.

\section{Three Phase ac Railgun}

The three phase ac railgun system (fig. 11) offers an interesting alternative to the two phase system. The three phase system uses a three rail railgun. Since the three phase currents sum to zero, the return path is intrinsic.

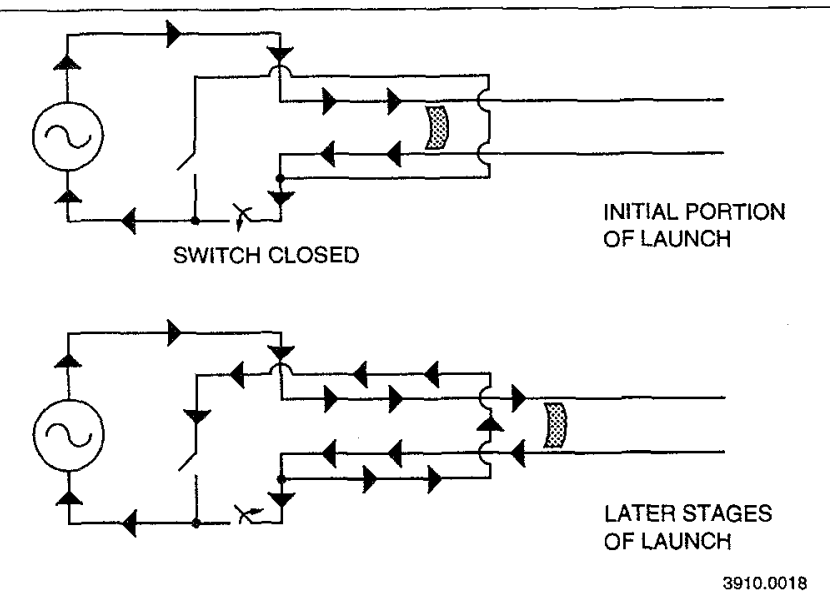

Figure 10. AC drive system active railgun impedance control

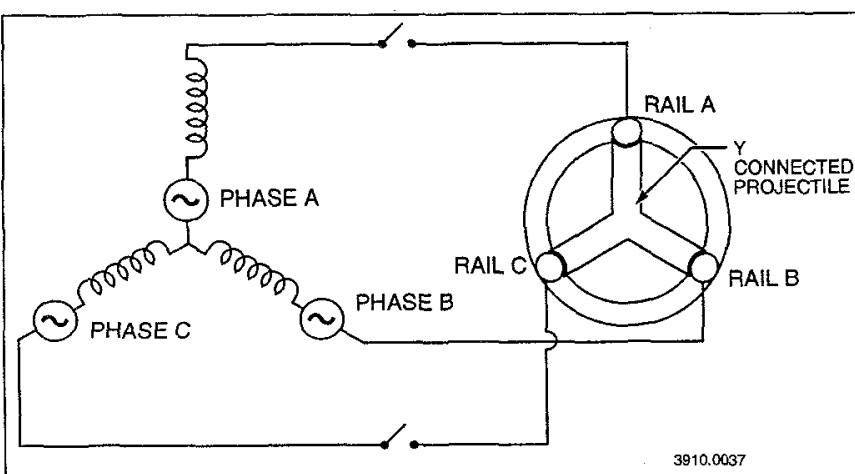

Figure 11. Three phase, ac railgun system

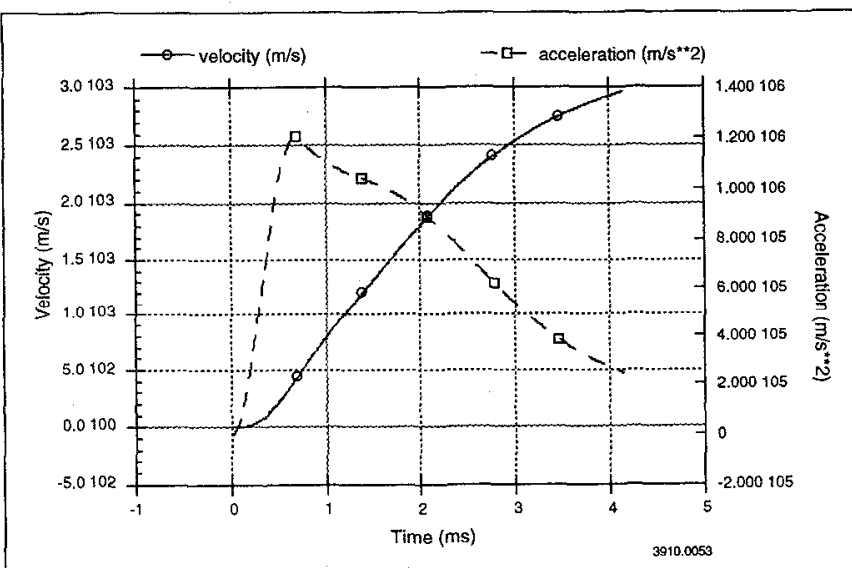

Figure 12. Acceleration and velocity profile for a three phase ac railgun system
For a balanced three phase system, the currents can be described as,

$$
\begin{aligned}
& I_{a}=I_{m} \sin (\omega t) \\
& I_{b}=I_{m} \sin \left(\omega t-120^{\circ}\right) \\
& I_{c}=I_{m} \sin \left(\omega t-240^{\circ}\right)
\end{aligned}
$$

where

$$
\mathrm{I}_{\mathrm{m}}=\text { peak current }
$$

The force on the projectile can be given as

$$
\mathrm{F}=\frac{3}{4} \mathrm{~L}_{\mathrm{e}}^{\prime} \mathrm{I}_{\mathrm{m}}^{2}=\frac{\mathrm{L}_{\mathrm{e}}^{\prime}}{2}\left(\mathrm{I}_{\mathrm{a}}^{2}+\mathrm{I}_{\mathrm{b}}^{2}+\mathrm{I}_{\mathrm{c}}^{2}\right)
$$

where

$\mathrm{L}_{\mathrm{e}}=$ effective inductance gradient per phase (explained below)

In the three phase railgun the mutual inductance between the rails reduces the magnetic signature of the gun. Also the mutual inductance between the rails " compensates" the self-inductance of the rails leading to a lower driving L'. For the same peak current and L', the force on the projectile in a three phase gun is higher than the force in the two phase gun. Hence it appears that the three phase ac system does not require the compensating rails (or the anti-augmenting rails) of the two phase system.

Let us compute the flux linkage $\psi_{\mathrm{a}}$ of phase a of the three phase railgun system,

$$
\begin{aligned}
\Psi_{\mathrm{a}} & =\mathrm{L}_{\mathrm{a}} \mathrm{I}_{\mathrm{a}}+\mathrm{MI}_{\mathrm{b}}+\mathrm{MI}_{\mathrm{c}} \\
& =\mathrm{L}_{\mathrm{a}} \mathrm{I}_{\mathrm{a}}+\mathrm{M}\left(\mathrm{I}_{\mathrm{b}}+\mathrm{I}_{\mathrm{c}}\right) \\
& =\left(\mathrm{L}_{\mathrm{a}}-\mathrm{M}\right) \mathrm{I}_{\mathrm{a}} \\
\mathrm{L}_{\mathrm{e}} & =\left(\mathrm{L}_{\mathrm{a}}-\mathrm{M}\right)
\end{aligned}
$$

where

$\mathrm{L}_{\mathrm{a}}=$ self inductance of phase a

$\mathrm{M}=$ mutual inductance between the phases

In case of the three phase ac railgun, the locus of the center of force on the projectile is a circle. This leads to a non-uniform loading on the projectile and is highly undesirable.

When an ac power supply is connected to a load, a dc transient appears in the current, this destroys the symmetry of the sinusoidal current and results in distortion of the acceleration profile in case 


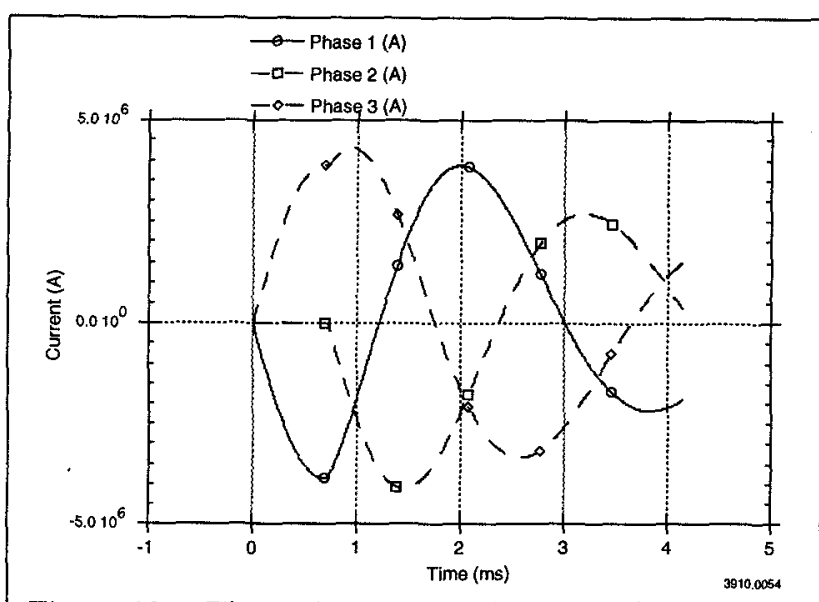

Figure 13. Three phase current in an ac railgun system

of the multiphase ac railgun. The dc transient can be minimized by switching in each phase at peak voltage. In case of the three phase system, switching in each phase at peak voltage would require a neutral connection or rail as a return path. However, this need for the neutral connection can be eliminated by switching two phases simultaneously at the peak line to line voltage (fig. 11) and the third phase is switched in at its peak voltage creating a balanced three phase system. Figures 12 and 13 show the acceleration profile and the phase currents for a three phase ac railgun system.

\section{Operating Modes Conclusions}

The three phase staged discharge mode offers improved performance (a high acceleration ratio). The selected mode drives the gun with a dc pulse and hence requires a lower operating voltage than the ac railgun system. The two phase ac railgun system requires an extra set of rails (anti-augmenting rails) to achieve the desired acceleration ratio. This implies higher losses in the two phase ac system. In general the ac system requires more reactive power than the staged discharge system or the single phase system because the magnetic energy is oscillating in and out of the railgun during each cycle. This also implies that for the same electrical frequency the ac system requires higher voltage than the staged discharge system. Hence the upper limit on the electrical frequency of operation is lower in case of the ac railgun systems. The three phase ac railgun system also has the additional problem of an eccentric force which is not favorable from the interior ballistics view point. The single phase system has the problem of poor control and flexibility and also higher machine mass. The three phase staged discharge system has better features from a control and flexibility stand point than the two phase staged discharge system.

\section{References}

1. M.L. Spann, et al, "Compulsator Research at The University of Texas at Austin -- An Overview," IEEE Transactions on Magnetics, vol 25, no. 1, January 1989, pp 529-537.

2. S.B. Pratap, et al, "Future Trends for Compulsators Driving Railguns," IEEE Transactions on Magnetics, Vol. Mag-22, No. 6, pp 1681-1683, November 1986.

3. S.B. Pratap, et al, "Advanced Compulsators for Railguns," IEEE Transactions on Magnetics, Vol. 25, No. 1, January. 1989.

4. M.D. Driga, et al, "Advanced Compulsator Design," IEEE Transactions on Magnetics, Vol. 25 No. 1, January 1989. 